\title{
Regioselective Oxidation of 4,5-Disubstituted Pyrazoles: Controlled by Resonance Stabilization versus Hyperconjugation
}

\author{
Ka Young Lee, Seung Chan Kim, and Jae Nyoung Kim* \\ Department of Chemistry and Institute of Basic Science, Chonnam National University, Gwangju 500-575, Korea \\ *E-mail:kimjn@chonnam.ac.kr \\ Received August 8, 2005
}

Key Words : Pyrazole, Hyperconjugation, Resonance, DDQ oxidation

DDQ (2,3-dichloro-5,6-dicyano-1,4-benzoquinone) is one of the most frequently used reagents to perform several organic transformations such as dehydrogenation ${ }^{1 \mathrm{a}}$ or oxidation of allylic and benzylic alcohols. ${ }^{\mathrm{b}}$ DDQ also has been used for the deprotection of several protecting groups from alcohols such as allyl, p-methoxybenzyl (PMB), and dimethoxy benzyl (DMB) groups. ${ }^{\text {1c-e }}$

Recently, we reported the synthesis of polysubstituted pyrazoles $^{2 a}$ and oxidation of the pyrazoles into indazoles by DDQ oxidation. ${ }^{2 b}$ During the oxidation of pyrazole 1a with DDQ ${ }^{2 b}$ we observed an interesting regioselective formation of $2 \mathbf{a}(58 \%)$ by the moisture in the reaction medium (Scheme 1). In the reaction we could not find other regioisomeric alcohol derivatives. Intrigued by the selective formation of 2a we intended to examine the selective oxidation of pyrazole derivatives with DDQ. In order to increase the yields of oxygenated products we used carboxylic acids and wish to report herein the results.

For the mechanism of DDQ oxidations, initial rate determining hydride abstraction by DDQ from the substrate to form the carbocation intermediate is generally accepted..$^{3-5}$ Following proton transfer from the carbocation leading to hydroquinone formation is a rapid process. ${ }^{3-5}$ Thus, dehydrogenation is dependent upon the degree of stabilization of the incipient carbocation and is enhanced by the presence of functionality capable of stabilizing the transition state.
The carbon atom of the carbocation (I) is positioned at the $\alpha$-position of an enamine-like moiety of pyrazole ring, while the carbon atom of the carbocation (II) is positioned nearby the electron-withdrawing imine functionality (Scheme 1). In addition, carbocation (I) can be stabilized further by the resonance effect of the phenyl group at the 3-position of pyrazole ring. Thus, when we consider the resonance stabilization effects, selective formation of carbocation (I) and $\mathbf{2 a}$, as a result, could be easily understood.

In order to examine the generality of this selectivity we examined the oxidation of pyrazoles 1a-d with DDQ in the presence of carboxylic acid as the carbocation quencher. As expected pyrazole $\mathbf{1 a}$ was transformed into $\mathbf{2 b}(62 \%)$ with acetic acid (Scheme 1 and entry 1 in Table 1). We did not detect nor isolate the other possible regioisomer. This was true for most of the other cases as shown in Table 1 (entries 2-5) irrespective of the kinds of carboxylic acids. However, when we carried out the reaction with $\mathbf{1 d}$, we obtained $\mathbf{2} \mathbf{g}^{\mathbf{\prime}}$ as the major product instead of $\mathbf{2 g}$ (entry 6). The two compounds $\mathbf{2} \mathbf{g}$ and $\mathbf{2} \mathbf{g}$ ' were very difficult to separate and we determined the ratios $\left(\mathbf{2} \mathbf{g} / \mathbf{2} \mathbf{g}^{\prime}=1: 4\right)$ based on ${ }^{1} \mathrm{H}$ NMR spectrum of the mixtures (see, Experimental section). With propionic acid and $\mathbf{1 d}$, we obtained a mixtures of $\mathbf{2} \mathbf{h}$ and $\mathbf{2} \mathbf{h}^{\prime}$ in a ratio of $1: 12$ (entry 7). Fortunately, the major isomer $\mathbf{2} \mathbf{h}^{\prime}$ could be isolated in pure state after determination of the ratios in this case. The results can be easily explained when

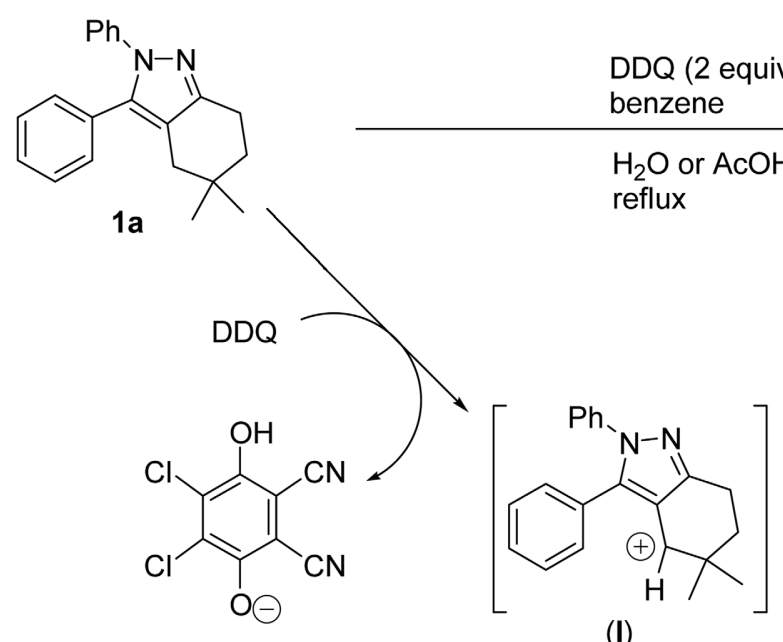

(I)<smiles>[R6]C1c2c(nn(-c3ccccc3)c2-c2ccccc2)CCC1(C)C</smiles>

2a: $\mathrm{R}=\mathrm{H}(58 \%)^{2 \mathrm{~b}}$

2b: $\mathrm{R}=\mathrm{COCH}_{3}(62 \%)$

Scheme 1 
Table 1. Selective functionalization of pyrazole derivatives

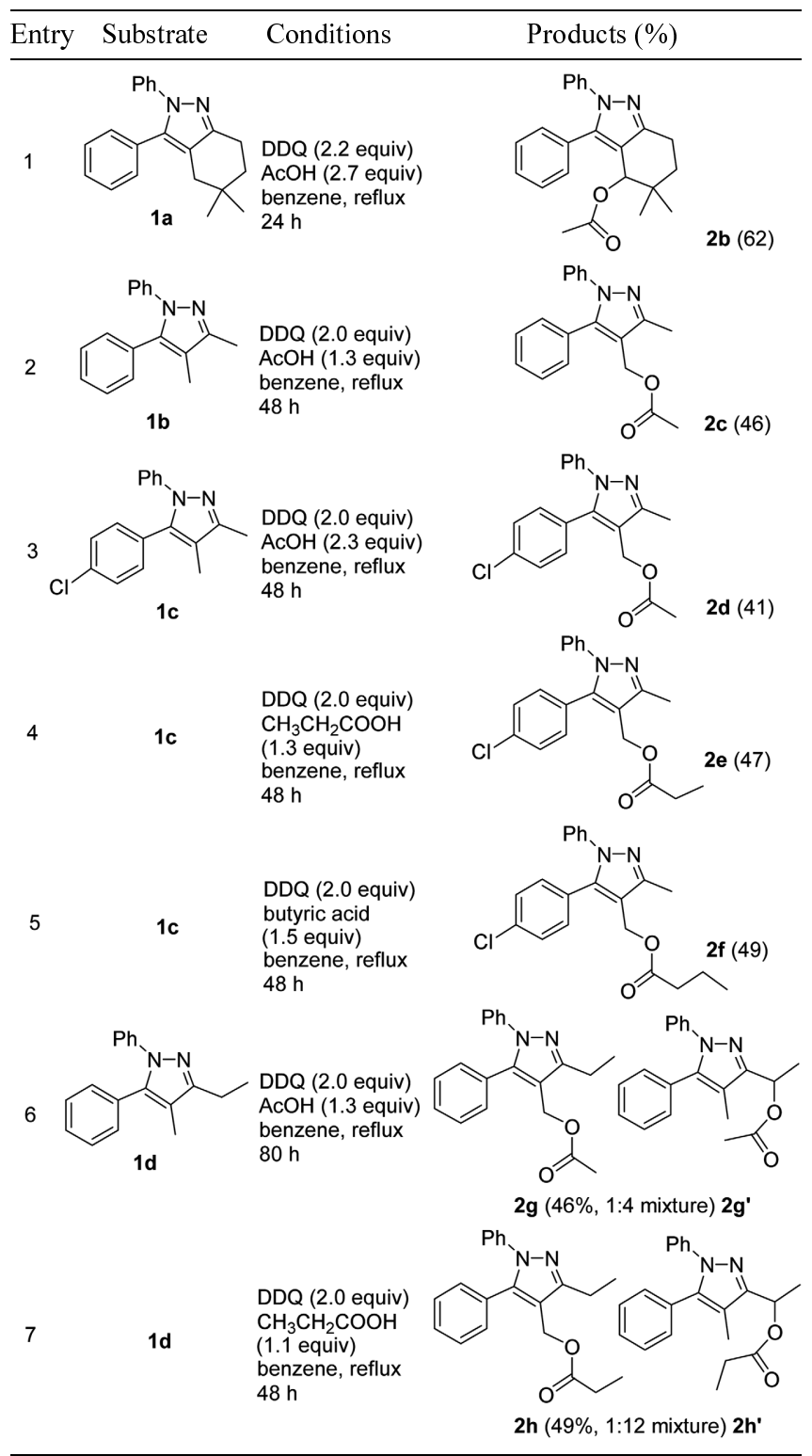<smiles>CC1(C)CCc2nn(-c3ccccc3)c(-c3ccccc3)c2C1=O</smiles><smiles>Cc1c(C=O)nn(-c2ccccc2)c1-c1ccccc1</smiles>

$\mathrm{C}^{+}$intermediate of $1 \mathrm{a}$

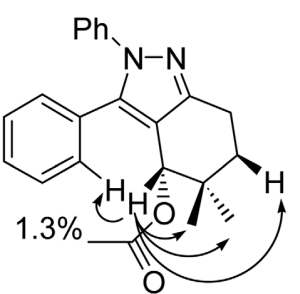

$2.4 \%$
$1.4 \%$
$0.5 \%$

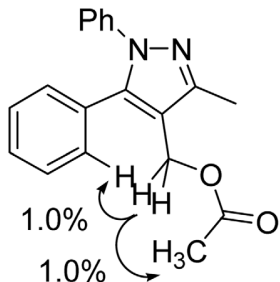

Figure 2. NOE results of $\mathbf{2} \mathbf{b}$ and $\mathbf{2 c}$.

stabilized via the hyperconjugation effect with the $\mathrm{C}-\mathrm{CH}_{3} \sigma$ bond. The structures of products $\mathbf{2} \mathbf{b}-\mathbf{h}^{\prime}$ ' were identified with the IR, ${ }^{1} \mathrm{H}$ and ${ }^{13} \mathrm{C}$ NMR, NOE (Figure 2), and/or mass data.

In summary, we prepared some functionalized pyrazoles by the selective formation of carbocation intermediate with DDQ and the following reaction with carboxylic acids. In the oxidation, resonance stabilization and hyperconjugative stabilization effects played important roles for the selective formation of the more stable carbocation.

\section{Experimental Section}

Typical procedure for the synthesis of $2 \mathrm{~b}$ : A stirred mixture of 1a $(151 \mathrm{mg}, 0.5 \mathrm{mmol})$, DDQ (250 mg, 1.1 $\mathrm{mmol})$, and $\mathrm{AcOH}(81 \mathrm{mg}, 1.35 \mathrm{mmol})$ in benzene $(5 \mathrm{~mL})$ was heated to reflux for $24 \mathrm{~h}$. After removal of the solvent and column chromatographic purification process (hexanes/ ether, $8: 1)$ we obtained pure $\mathbf{2 b}$ as clear oil, $112 \mathrm{mg}(62 \%)$. Compounds $\mathbf{2 c - h}$ ' were synthesized similarly and the spectroscopic data are as follows.

Compound 2b: 62\%; oil; IR (KBr) 1732, 1504, $1369 \mathrm{~cm}^{-1}$; ${ }^{1} \mathrm{H}$ NMR (300 MHz, $\left.\mathrm{CDCl}_{3}\right) \delta 0.99(\mathrm{~s}, 3 \mathrm{H}), 1.03(\mathrm{~s}, 3 \mathrm{H})$, 1.59-1.68 (m, 1H), $1.74(\mathrm{~s}, 3 \mathrm{H}), 1.91-2.01(\mathrm{~m}, 1 \mathrm{H}), 2.73-$ $2.95(\mathrm{~m}, 2 \mathrm{H}), 5.83(\mathrm{~s}, 1 \mathrm{H}), 7.11-7.14(\mathrm{~m}, 2 \mathrm{H}), 7.19-7.33(\mathrm{~m}$, $8 \mathrm{H}) ;{ }^{13} \mathrm{C}$ NMR $\left(75 \mathrm{MHz}, \mathrm{CDCl}_{3}\right) \delta 19.94,20.68,24.17$, $24.47,32.43,34.89,71.38,116.05,124.74,126.89,128.37$, $128.43,128.66,129.51,129.92,139.86,141.16,149.69$, 170.15; ESIMS $m / z 361\left(\mathrm{M}^{+}+\mathrm{H}\right)$.

Compound 2c: 46\%; oil; IR (KBr) 2958, 2931, 1739 , $1597,1504,1381,1230 \mathrm{~cm}^{-1}$; ${ }^{1} \mathrm{H} \mathrm{NMR}\left(300 \mathrm{MHz} \mathrm{CDCl}_{3}\right) \delta$ 2.09 (s, 3H), 2.41 (s, 3H), 4.97 (s, 2H), 7.17-7.36 (m, 10H); ${ }^{13} \mathrm{C}$ NMR $\left(75 \mathrm{MHz}, \mathrm{CDCl}_{3}\right) \delta 11.93,20.99,57.05,113.96$, $124.78,126.99,128.54,128.63,128.73,129.50,129.79$, 139.69, 143.06, 149.53, 170.93; ESIMS $m / z 307\left(\mathrm{M}^{+}+\mathrm{H}\right)$.

Compound 2d: 41\%; oil; IR (KBr) 2920, 1739, 1597, 1504, 1377, $1230 \mathrm{~cm}^{-1}$; ${ }^{1} \mathrm{H}$ NMR $\left(300 \mathrm{MHz}, \mathrm{CDCl}_{3}\right) \delta 2.09$ (s, 3H), 2.41 (s, 3H), $4.95(\mathrm{~s}, 2 \mathrm{H}), 7.12-7.35(\mathrm{~m}, 9 \mathrm{H}) ;{ }^{13} \mathrm{C}$ NMR $\left(75 \mathrm{MHz}, \mathrm{CDCl}_{3}\right) \delta 11.94,21.01,56.87,114.24$, $124.88,127.29,128.01,128.94$ (2C), 131.08, 134.91, 139.49, 141.85, 149.71, 170.88; ESIMS $m / z 341\left(\mathrm{M}^{+}+\mathrm{H}\right)$, $343\left(\mathrm{M}^{+}+2+\mathrm{H}\right)$.

Compound 2e: 47\%; oil; IR (KBr) 2978, 2939, 1736, $1597,1504,1377 \mathrm{~cm}^{-1} ;{ }^{1} \mathrm{H}$ NMR $\left(300 \mathrm{MHz}, \mathrm{CDCl}_{3}\right) \delta 1.16$ (t, $J=7.5 \mathrm{~Hz}, 3 \mathrm{H}), 2.36(\mathrm{q}, J=7.5 \mathrm{~Hz}, 2 \mathrm{H}), 2.41(\mathrm{~s}, 3 \mathrm{H})$, 4.95 (s, 2H), 7.13-7.34 (m, 9H); $\left.{ }^{13} \mathrm{C} \mathrm{NMR} \mathrm{(75} \mathrm{MHz,} \mathrm{CDCl}_{3}\right)$ $\delta 9.11,11.93,27.58,56.74,114.35,124.83,127.24,128.01$, 128.89 (2C), 131.05, 134.85, 139.46, 141.76, 149.67, surpasses the resonance stabilization effect in these cases. depicted in Figure 1, the carbocation of 1 a can also be

we consider the hyperconjugative stabilization effect. ${ }^{6,7}$ The corresponding carbocation of $\mathbf{1 d}$ (Figure 1) can be stabilized by hyperconjugation with the $\mathrm{C}-\mathrm{H}$ s-bond as shown. From the results of selective formation of $\mathbf{2} \mathbf{g}^{\prime}$ over $\mathbf{2} \mathbf{g}$ and $\mathbf{2} \mathbf{h}^{\prime}$ over $\mathbf{2 h}$, we could conclude that the hyperconjugation effect 
174.25; ESIMS $m / z 355\left(\mathrm{M}^{+}+\mathrm{H}\right), 357\left(\mathrm{M}^{+}+2+\mathrm{H}\right)$.

Compound 2f: 49\%; oil; IR (KBr) 2966, 2931, 1736, 1597, 1504, $1381 \mathrm{~cm}^{-1} ;{ }^{1} \mathrm{H}$ NMR (300 MHz, $\left.\mathrm{CDCl}_{3}\right) \delta 0.96$ (t, $J=7.5 \mathrm{~Hz}, 3 \mathrm{H}), 1.67$ (sextet, $2 \mathrm{H}), 2.31(\mathrm{t}, J=7.5 \mathrm{~Hz}$, 2H), 2.40 (s, 3H), 4.95 (s, 2H), 7.12-7.34 (m, 9H); ${ }^{13} \mathrm{C} \mathrm{NMR}$ $\left(75 \mathrm{MHz}, \mathrm{CDCl}_{3}\right) \delta 11.91,13.63,18.47,36.21,56.65$, $114.35,124.84,127.25,128.02,128.90$ (2C), 131.04, 134.85, 139.47, 141.78, 149.67, 173.48; ESIMS m/z 369 $\left(\mathrm{M}^{+}+\mathrm{H}\right), 371\left(\mathrm{M}^{+}+2+\mathrm{H}\right)$.

Compounds $\mathbf{2 g}$ and $\mathbf{2} \mathbf{g}$ ' were separated together in a ratio of 1 : 4 (based on ${ }^{1} \mathrm{H}$ NMR) in $46 \%$ yield. IR and mass spectra were taken as a mixture: IR (KBr) 2981, 2931, 1736, 1597, 1504, 1373, $1242 \mathrm{~cm}^{-1}$; ESIMS m/z $321.2\left(\mathrm{M}^{+}+\mathrm{H}\right)$.

Compound 2g: ${ }^{1} \mathrm{H}$ NMR (300 MHz, $\left.\mathrm{CDCl}_{3}\right) \delta 1.36(\mathrm{t}, J=$ $7.5 \mathrm{~Hz}, 3 \mathrm{H}), 2.08$ (s, 3H), 2.80 (q, $J=7.5 \mathrm{~Hz}, 2 \mathrm{H}), 4.97$ (s, 2H), 7.13-7.37 (m, 10H).

Compound 2g': ${ }^{1} \mathrm{H}$ NMR (300 MHz, $\left.\mathrm{CDCl}_{3}\right) \delta 1.73(\mathrm{~d}, J=$ $6.6 \mathrm{~Hz}, 3 \mathrm{H}), 2.10(\mathrm{~s}, 3 \mathrm{H}), 2.11(\mathrm{~s}, 3 \mathrm{H}), 6.15(\mathrm{q}, J=6.6 \mathrm{~Hz}$, $1 \mathrm{H}), 7.13-7.37$ (m, 10H).

Compounds $\mathbf{2 h}$ and $\mathbf{2} \mathbf{h}$ ' were separated together in a ratio of 1 : 12 (based on ${ }^{1} \mathrm{H}$ NMR) in 49\% yield. Pure $2 \mathbf{h}^{\prime}$ was isolated by column chromatography after checking the ratio of $2 \mathbf{h} / \mathbf{2 h}$ '.

Compound 2h: ${ }^{1} \mathrm{H}$ NMR (300 MHz, $\left.\mathrm{CDCl}_{3}\right) \delta 1.16$ (t, $J=$ $7.5 \mathrm{~Hz}, 3 \mathrm{H}), 1.35$ (t, $J=7.5 \mathrm{~Hz}, 3 \mathrm{H}), 2.36(\mathrm{q}, J=7.5 \mathrm{~Hz}$, 2H), 2.80 (q, $J=7.5 \mathrm{~Hz}, 2 \mathrm{H}), 4.97$ (s, 2H), 7.14-7.35 (m, $10 \mathrm{H})$.

Compound 2h': oil; IR (KBr) 2985, 2931, 1736, 1597, $1504,1373,1188 \mathrm{~cm}^{-1} ;{ }^{1} \mathrm{H}$ NMR $\left(300 \mathrm{MHz}, \mathrm{CDCl}_{3}\right) \delta 1.18$ $(\mathrm{t}, J=7.5 \mathrm{~Hz}, 3 \mathrm{H}), 1.72(\mathrm{~d}, J=6.6 \mathrm{~Hz}, 3 \mathrm{H}), 2.10(\mathrm{~s}, 3 \mathrm{H})$, 2.40 (q, $J=7.5 \mathrm{~Hz}, 2 \mathrm{H}), 6.16(\mathrm{q}, J=6.6 \mathrm{~Hz}, 1 \mathrm{H}), 7.14-7.35$ $(\mathrm{m}, 10 \mathrm{H}) ;{ }^{13} \mathrm{C} \mathrm{NMR}\left(75 \mathrm{MHz}, \mathrm{CDCl}_{3}\right) \delta 8.47,9.16,19.33$, $27.78,66.33,114.38,124.77,126.77,128.08,128.40$, 128.63, 129.93, 130.44, 140.03, 140.89, 150.82, 173.91; ESIMS $m / z 335\left(\mathrm{M}^{+}+\mathrm{H}\right)$.

Acknowledgments. This work was supported by the grant (R-05-2003-000-10042-0) from the Basic Research Program of the Korea Science and Engineering Foundation (Now controlled under the authority of Korea Research Foundation). Spectroscopic data was obtained from the Korea Basic Science Institute, Gwangju branch.

\section{References and Notes}

1. (a) Fu, P. P.; Harvey, R. G. Chem. Rev. 1978, 78, 317. (b) Becker, H.-D.; Bjork, A.; Alder, E. J. Org. Chem. 1980, 45, 1596. (c) Walker, D.; Hiebert, J. D. Chem. Rev. 1967, 67, 153. (d) Horita, K.; Yoshioka, T.; Tanaka, T.; Oikiwa, Y.; Yonemitsu, O. Tetrahedron 1986, 42, 3021. (e) Greene, T. W.; Wuts, P. G. M. Protective Groups in Organic Synthesis, $3^{\text {rd }}$ ed; John Wiley \& Sons: New York, 1999.

2. (a) Lee, K. Y.; Kim, J. M.; Kim, J. N. Tetrahedron Lett. 2003, 44, 6737. (b) Lee, K. Y.; Gowrisankar, S.; Kim, J. N. Tetrahedron Lett. 2005, 46, 5387.

3. (a) Muller, P.; Rocek, J. J. Am. Chem. Soc. 1972, 94, 2716. (b) Stoos, F.; Rocek, J. J. Am. Chem. Soc. 1972, 94, 2719. (c) Thummel, R. P.; Cravey, W. E.; Cantu, D. B. J. Org. Chem. 1980, 45, 1633. (d) Wurche, F.; Sicking, W.; Sustmann, R.; Klarner, F.G.; Ruchardt, C. Chem. Eur. J. 2004, 10, 2707 and further references cited therein.

4. For hydroxylation and related oxidations with DDQ, see (a) Xu, Y.-C.; Lebeau, E.; Gillard, J. W.; Attardo, G. Tetrahedron Lett. 1993, 34, 3841. (b) Ramdayal, F. D.; Kiemle, D. J.; LaLonde, R. T. J. Org. Chem. 1999, 64, 4607. (c) Zhang, Z.; Magnusson, G. J. Org. Chem. 1996, 61, 2394. (d) Corey, E. J.; Xiang, Y. B. Tetrahedron Lett. 1987, 28, 5403. (e) Tanemura, K.; Suzuki, T.; Nishida, Y.; Satsumabayashi, K.; Horaguchi, T. J. Chem. Soc., Perkin Trans. 1 2001, 3230.

5. Further references of DDQ oxidations, see (a) Peng, K.; Chen, F.; She, X.; Yang, C.; Cui, Y.; Pan, X. Tetrahedron Lett. 2005, 46, 1217. (b) Flogel, O.; Reibig, H.-U. Eur. J. Org. Chem. 2004, 2797. (c) Pinho e Melo, T. M. V. D.; Cabral, A. M. T. D. P. V.; Rocha Gonsalves, A. M. d'A.; Beja, A. M.; Paixao, J. A.; Silva, M. R.; Alte da Veiga, L.; Gilchrist, T. L. J. Org. Chem. 1999, 64, 7229. (d) Wallace, D. J.; Gibb, A. D.; Cottrell, I. F.; Kennedy, D. J.; Brands, K. M. J.; Dolling, U. F. Synthesis 2001, 1784. (e) Jevric, M.; Taylor, D. K.; Greatrex, B. W.; Tiekink, E. R. T. Tetrahedron 2005, 61, 1885. (f) Haldar, P.; Guin, J.; Ray, J. K. Tetrahedron Lett. 2005, 46, 1071. (g) Cheruku, S. R.; Padmanilayam, M. P.; Vennerstrom, J. L. Tetrahedron Lett. 2003, 44, 3701. (h) Ying, B.-P.; Trogden, B. G.; Kohlman, D. T.; Liang, S. X.; Xu, Y.-C. Org. Lett. 2004, 6, 1523. (i) Minuti, L.; Taticchi, A.; Marrocchi, A.; Lanari, D.; Gacs-Baitz, E.; Gomory, A. Tetrahedron Lett. 2005, 46, 949.

6. (a) Laube, T. Acc. Chem. Res. 1995, 28, 399. (b) Tostes, J. R.; Seidl, P. R.; Taft, C. A.; Lie, S. K.; Carneiro, J. W. de M.; Brown, W.; Lester Jr, W. A. Theochem 1996, 388, 85.

7. Further readings on hyperconjugations, see (a) Alabugin, I. V.; Manoharan, M. J. Org. Chem. 2004, 69, 9011. (b) Muller, T.; Margraf, D.; Syha, Y. J. Am. Chem. Soc. 2005, 127, 10852. (c) Schormann, M.; Garratt, S.; Hughes, D. L.; Green, J. C.; Bochmann, M. J. Am. Chem. Soc. 2002, 124, 11266. (d) Brandt, P.; Jia, Z. S.; Thibblin, A. J. Org. Chem. 2002, 67, 7676. 